\title{
UN MODELO DE LIDERAZGO PARA LA RETENCIÓN DE ENFERMEROS/AS PROFESIONALES
}

\section{A leadership model for the retention of professional nurses}

\section{Marina Olmos}

Recibido: 27/08/2021 - Aceptado: 26/10/2021

\section{RESUMEN:}

En el ámbito de Recursos Humanos es escaso el conocimiento sobre la implicancia de las características del fenómeno del Liderazgo en la retención del talento, en lo referido particularmente a la población de enfermeros/as profesionales. Este estudio empírico basado en un diseño no experimental de alcance descriptivo, tuvo por objetivo general evaluar si existe relación entre las estrategias de intervención enfocadas en el equipo de trabajo que desarrollan los Jefes de Unidad de Enfermería, y la retención del recurso humano de enfermería del Hospital Privado de Comunidad de la ciudad de Mar del Plata (Argentina), para generar una propuesta competitiva y sostenible de retención de Recursos Humanos basada en un modelo de liderazgo ajustado a las necesidades de los colaboradores.

\begin{abstract}
In the field of Human Resources, there is little knowledge about the implication of the characteristics of the Leadership phenomenon in the retention of talent, particularly in relation to the population of professional nurses. This empirical study based on a non-experimental design with a descriptive scope, had the general objective of evaluating whether there is a relationship between the intervention strategies focused on the work team developed by the Heads of Nursing Unit, and the retention of the nursing human resource of the Private Community Hospital of the city of Mar del Plata (Argentina), to generate a competitive and sustainable proposal for the retention of Human Resources based on a leadership model adjusted to the needs of employees.
\end{abstract}

PALABRAS CLAVE: liderazgo, gestión del desempeño, competencias, retención del talento, enfermero/a profesional.

KEYWORDS: leadership, performance management, competences, talent retention, professional nurse.

\footnotetext{
${ }^{1}$ Magíster en Dirección y Gestión de Recursos Humanos. Universidad Blas Pascal, Córdoba, Argentina. Licenciada en Psicología. Universidad Nacional de Mar del Plata, Buenos Aires, Argentina.

Especialista en selección de personas, en particular puestos de liderazgo; en salud laboral, gestión del desempeño y desarrollo de las personas y empresas. Consultoría y asesoramiento a emprendedores y pymes en Gestión Humana y planificación estratégica. Orientación vocacional y laboral. Asesoramiento a jóvenes profesionales. marinacandelaolmos@gmail.com
} 


\section{Introducción}

Las organizaciones del rubro Salud a nivel mundial se ven afectadas cíclicamente por la escasez de enfermeros/as, quienes constituyen el grupo más numeroso de dispensadores de atención de salud siendo que sus servicios son esenciales para la prestación de cuidados seguros y eficaces. Por cual, podemos definir al puesto de enfermeros/as como crítico y clave para las Instituciones de Salud.

La presente investigación, ejemplifica la problemática mencionada en una empresa privada del rubro Salud de la ciudad de Mar del Plata.

En simultáneo, en la actualidad nos encontramos inmersos en un mundo VICA (volátil, incierto, complejo y ambiguo). Podemos decir que, este contexto impulsó en la gestión de Recursos Humanos el cambio del paradigma centrado en los resultados a una gestión centrada en las personas. Este enfoque considera a las personas como creadoras de valor, potenciales a desarrollar y como claves del éxito organizacional. En este contexto, el desafío hoy para las organizaciones del rubro Salud es lograr una eficiente gestión de sus talentos como generador de ventajas competitivas sostenibles. Desde esta perspectiva, el presente estudio se propuso brindar una solución integral enfocada en generar estrategias de retención de los recursos valiosos de enfermería, haciendo énfasis en el rol fundamental que ejerce el Liderazgo en el capital humano.

\section{Liderazgo}

El fenómeno del Liderazgo es un tema que ha sido estudiado ampliamente por variedad de disciplinas y autores, por cual posee definiciones y niveles de análisis diversos. A continuación, se detalla un recorrido del concepto desde sus inicios hasta la actualidad.

En una primera etapa, las investigaciones se centraron en identificar las cualidades personales inherentes que los individuos poseían para ser considerados como líderes exitosos. Luego, se enfocaron en el estudio de los comportamientos que los líderes demostra- ban y se añadieron estudios, relacionados con variables situacionales o de contingencia que moderan la relación comportamientoresultado del liderazgo. Luego, se sumaron estudios que buscaban asociar la visión del liderazgo exitoso a la competencia de eficacia. Podemos decir que, se pensaba el liderazgo en términos de resultados; así como, una relación asimétrica entre líder y subordinado basada en la orientación a logros y productividad. En estas etapas, los valores asociados eran el compromiso y la responsabilidad. En un principio, las investigaciones vincularon mayormente el liderazgo a factores intrínsecos al individuo. Aunque, luego la mirada se amplió incluyéndose los factores extrínsecos y el impacto de estos en el liderazgo. Desde esta perspectiva, se plantean las siguientes teorías:

- Teoría de los rasgos

- Teorías del comportamiento

- Teorías de contingencia sobre el liderazgo

- La eficacia y el liderazgo eficaz

Luego, el fenómeno del liderazgo se desarroIló desde enfoques contemporáneos. En el mundo VICA actual (volátil, incierto, complejo y ambiguo) una gestión eficiente requiere en los líderes el desarrollo de nuevas competencias. Podemos decir que este contexto trajo consigo el cambio del paradigma centrado en los resultados, a una gestión centrada en las personas. Estos tiempos nos invitan a construir colectivamente.

El líder se sustenta de metodologías efectivas, integrales y con un abordaje estratégico bajo una mirada de sustentabilidad organizacional. En la actualidad, el líder se posiciona como promotor del bienestar y desarrollo de las personas, con capacidad de escucha activa, ofreciendo su ayuda y apoyo al otro. Asimismo, emergen líderes con habilidades comunicacionales de tipo asertivas, enfocados en comunicar con claridad las expectativas y objetivos a sus equipos, brindando retroalimentación continua y constructiva; líderes dispuestos a delegar y alentar la cooperación, promoviendo espacios de diálogo compartidos y consenso de las modalidades de trabajo; líderes con actitud resiliente fren- 
te a los contextos adversos y cambiantes, que se enfocan en los logros más que en los resultados.

Desde este enfoque, pensamos al empleado en términos de colaborador siendo este un capital humano fundamental; consideramos a las personas como creadoras de valor, potenciales a desarrollar y como claves del éxito organizacional.

Hoy día, los valores que se difunden son el trabajo en equipo, la innovación, la integridad y el desarrollo.

Las teorías que encuadran en este enfoque son las siguientes:

- Liderazgo transformacional- transaccional

- Liderazgo carismático-visionario

- Liderazgo de equipos

- Liderazgo de servicio

- Liderazgo y efectividad

- Liderazgo en tiempos actuales

La presente investigación, desarrolló el fenómeno del liderazgo de acuerdo con lo propuesto por los modelos del liderazgo transformacional, de servicio y a los enfoques de los tiempos actuales. De este modo, se alejó de la concepción tradicional de jefatura basada en la gestión verticalista y orientada netamente al cumplimiento de objetivos.

Siguiendo este lineamiento la investigación se encuadra en la concepción del Liderazgo Transformacional y de Servicio. Según Daft (2006) citado en Polifrone (2018), el liderazgo transformacional sirve para cambiar el statu quo, dado que articula, para los seguidores, los problemas en el sistema actual y una visión convincente de lo que podría ser una nueva organización. Por otro lado, los líderes transformacionales pueden provenir de distintos niveles de la organización. Por lo tanto, en una empresa puede haber muchos líderes de este tipo. El liderazgo transformacional lleva a los seguidores a convertirse en líderes. Los seguidores poseen mayor libertad para controlar su conducta. El líder transformacional agrupa a la gente entorno a una misión y define el marco dentro del cual los seguidores pueden operar con relativa libertad para alcanzar las metas de la organización. El líder transformacional despierta la conciencia de sus seguidores respecto a diversos problemas y asuntos, y ayuda a las personas a ver las cosas bajo distintas ópticas para así poder conseguir cambios en la producción o servicios. Asimismo, retomando las palabras de Polifrone (2018) este liderazgo está fundado en los valores, creencias y las cualidades personales del líder y no en un proceso de intercambio entre los líderes y los seguidores.

El liderazgo de servicio se relaciona con el liderazgo transformacional en tanto se enfocan en delegar autoridad en los seguidores para que ejerzan el liderazgo con el fin de alcanzar las metas de la organización. La teoría de liderazgo tradicional destaca la estructura entre líder y seguidor, en la cual este acepta la responsabilidad de parte del líder y es responsable ante él. Sin embargo, desde la óptica no tradicional del liderazgo se percibe al líder como un servidor de la gente y la organización. En el liderazgo de servicio se trata menos de dirigir o controlar, y más de concentrarse en ayudar a los seguidores a realizar su labor, y no en hacer que estos ayuden a los jefes a hacer la propia (Lussier y Achua, 2008, citado en Polifrone 2018).

\section{Competencias}

De acuerdo con Cravino (2018), las competencias son: "el conjunto de conocimientos habilidades, actitudes y valores que debe tener una persona para desempeñarse exitosamente en un puesto determinado, en una empresa determinada, en un contexto determinado y se encuentran definidos y descriptos mediante patrones de comportamiento observable".

Desde una aproximación más contemporánea Cravino (2018), define a la gestión de competencias, como la aplicación del modelo de competencias (documentación formal, habitualmente a través de un manual, de cuáles son las competencias de una organización) en una herramienta de gestión con tres finalidades principales: orientar la propia gestión de las personas; orientar la gestión de recursos humanos; orientar la gestión de los líderes. 
Por su parte, Cravino (2003) define a la gestión de Recursos Humanos basada en competencias como una metodología efectiva, integral, con un abordaje estratégico, que permite aumentar significativamente la satisfacción y el compromiso de las personas y produce resultados económicos eficientes bajo una mirada de sustentabilidad. Este enfoque considera a las personas como creadoras de valor, potenciales a desarrollar y como claves del éxito.

De este modo desde este abordaje actual, el modelo de gestión por competencias brinda coherencia y dirección a todas las acciones transversales de Recursos Humanos generando una visión compartida.

Desde esta perspectiva, es la gestión por competencias el vector conductor de los procesos de Recursos Humanos, representados en tres fases diferentes y consecutivas: flujo de ingreso (reclutamiento, selección, inducción y coaching), flujo interno (evaluación del desempeño, desarrollo, programas de capacitación, asesoramiento a Líderes, comunicación interna, clima laboral y beneficios) y flujo de salida (desvinculación, jubilaciones y renuncias).

Siguiendo la visión anteriormente expresada, las políticas del Departamento de Recursos Humanos del Hospital Privado de Comunidad de la ciudad de Mar del Plata (HPC) se enmarcan en la gestión basada en competencias. El Dpto. de RRHH se apoya en el concepto de competencias como aquellas características individuales de conocimiento, capacidad, aptitud o personalidad que influyen directamente en el rendimiento laboral de una persona.

\section{Caso Hospital Privado de Comunidad de la ciudad de Mar del Plata}

El presente trabajo estudió la población de enfermeros/as profesionales con diferentes niveles de formación académica del Hospital Privado de Comunidad de Mar del Plata. Esta Institución de Salud fue seleccionada dado que es la Institución médica privada de mayor complejidad de la ciudad y región, anualmente se realizan 664.247 consultas y 14.372 intervenciones quirúrgicas (Estadística anual Hospital Privado de Comunidad de Mar del Plata, 2019).

Desde la gestión de Recursos Humanos se observó una fuga de talentos. Se registró que entre el período de mayo 2018 a abril 2019 hubo 22 renuncias de enfermeros/ as con formación técnico profesional como también licenciados en enfermería y entre Abril 2019 a principios de Noviembre 2019 hubo 20 renuncias de enfermeros/as de diferentes niveles académicos.

Según el Organigrama, los profesionales de Enfermería reportan a los Jefes de Unidad. Si bien este término resulta un tanto obsoleto, en la actualidad la Organización está mutando hacia la mirada de la gestión por competencias, procesos, mejora continua y Visión de Líder.

Por su parte, es importante señalar que se observaron acciones aisladas de retención del talento gestionadas por el Departamento de Recursos Humanos del HPC aunque actualmente no posee formalmente un programa de políticas de retención del talento. A su vez, el mismo no se haya asociado a la política de remuneraciones de la empresa.

Por tanto, esta investigación se constituye como el punto de partida para visibilizar y concientizar acerca del lugar estratégico de las políticas de retención del talento siendo una herramienta eficiente en el marco del desarrollo sustentable organizacional.

Numerosos informes sugieren que las inversiones en la aplicación y mantenimiento de políticas y prácticas eficaces de gestión de los recursos humanos pueden contribuir de manera muy positiva a los resultados de la organización. La gestión eficaz de los recursos humanos puede estar relacionada con el personal y con los resultados de los cuidados en los pacientes (CIE, 2006).

A partir de lo expuesto se planteó como problema de investigación: ¿Existe relación entre las estrategias de intervención enfocadas en el equipo de trabajo que desarrollan los Jefes de Unidad de Enfermería y la retención 
del recurso humano de Enfermería del Hospital Privado de Comunidad de la ciudad de Mar del Plata?

\section{Objetivo general:}

El objetivo general consistió en determinar si existe relación entre las estrategias de intervención enfocadas en el equipo de trabajo que desarrollan los Jefes de Unidad de Enfermería y la retención del recurso humano de Enfermería del Hospital Privado de Comunidad de la ciudad de Mar del Plata.

\section{Objetivos específicos:}

Respecto a los objetivos específicos se buscó identificar las estrategias de intervención enfocadas en el equipo de trabajo que desarroIlan los Jefes de Unidad de Enfermería.

Definir la percepción del Liderazgo que desarrollan los Jefes de Unidad según los profesionales enfermeros/as del Hospital Privado de Comunidad de Mar del Plata.

Desarrollar una propuesta competitiva y sostenible de retención de Recursos Humanos basada en un modelo de Liderazgo ajustado a las necesidades de los colaboradores.

\section{Conclusiones}

En vista de los objetivos específicos 1 y 2 , podemos decir que los enfermeros/as perciben en los jefes un Liderazgo enfocado en los objetivos y resultados, acentuando como fortaleza el sólido conocimiento técnico necesario para orientarlos en su labor. Asimismo, identifican un líder receptivo frente a las sugerencias de mejora en los procesos de trabajo y destacan que les brinda la posibilidad de realizar la labor bajo criterios y experticia profesional. No obstante, los colaboradores depositan en los líderes otras expectativas y necesidades que hoy no están plenamente satisfechas. Partiendo de la premisa del objetivo general, podríamos inferir que esta relativa desconformidad en las intervenciones de los jefes impacta de manera desfavorable en el bienestar del enfermero/a, lo cual podría disminuir la intencionalidad de permanecer en el puesto de trabajo en el establecimiento actual. Por su parte, al analizar las entrevistas de los enfermero/as se detecta que ellos perciben el trabajo con sus compañeros como trabajo en equipo. Siguiendo la línea de Solorio (2012), el trabajo en equipo remite al conjunto de personas, con capacidades complementarias, que se organizan en forma determinada para lograr un objetivo común; refiere al conjunto de estrategias, acciones, técnicas y procedimientos que utiliza el grupo para alcanzar las metas.

En términos generales, se constata que los diferentes enfermero/as profesionales poseen marcadas expectativas sobre el accionar de su líder. Principalmente relacionadas con aspectos de la comunicación efectiva, escucha activa, gestión del desempeño, capacitación continua, desarrollo profesional, estimulación y motivación. A priori, es posible relacionar estas competencias y estrategias de gestión con los estilos de liderazgo configurados como contemporáneo, entre ellos se encuentran el Liderazgo transformacional, Liderazgo de servicio y líderes emocionalmente inteligentes.

Desde una perspectiva actual, Cravino (2003) expone que hoy se espera que los líderes ajusten su estilo a las características de sus colaboradores y también, asuman formas diferentes en función de la cambiante evolución contextual. Lo cual denomina líderes situacionales o líderes situacionales al cuadrado. De este modo, la competencia de adaptabilidad y flexibilidad asume un lugar vital en el rol del líder a fin de brindar respuestas ajustadas a las necesidades de los enfermero/as.

Partiendo de la mirada de Davis y Newstrom (2011) que plantean que el Liderazgo es un proceso de influencia, donde se influye en el comportamiento de los colaboradores hacia la consecución de logros y objetivos organizacionales, será a través de ese proceso que el enfermero/a se desarrolle y posicione como profesional, siendo por ello tan significativo para el colaborador las intervenciones que realice su líder. Podemos inducir que la autorrealización profesional de los enfermero/as se sustenta fuertemente en la satisfacción que les genera brindar calidad de atención al paciente y también, en el vínculo que posea y construya con su líder. En esta línea Robbins y Coulter (2009), visualizan a los 
líderes frente al reto de construir y reconstruir la confianza y la credibilidad de sus seguidores. Estos autores nos planten que los seguidores juzgan la credibilidad de un líder en términos de su honestidad, competencia y habilidad para inspirar.

Con relación al trabajo en equipo, si bien los enfermero/as subrayan que se desempeñan con equipos de trabajo basados en el compañerismo y colaboración, no se evidencian marcadas expectativas que pudieran impactar directamente en la entereza del profesional. Aunque destacan que prefieren un clima organizacional de tipo participativo, donde se pueda intercambiar conocimientos e ideas en todos los niveles de la Institución. Hoy consideran que es un desafío por desarrollar, a pesar de que la organización estructuralmente es un hospital escuela.

Otro dato de interés resulta del hecho que los resultados permiten presumir que para los Jefes de Unidad como para los enfermero/as, son igual de importantes las estrategias configuradas en relación a la gestión de la comunicación, del desempeño y desarrollo; como también, potenciar un clima organizacional de tipo participativo. En concordancia, los jefes están interesados en maximizar la comunicación entre ellos con el resto de los líderes de la estructura del Departamento de Enfermería en lo que refiere a rotación interna de colaboradores a fin de acompañar de modo eficiente al colaborador en ese proceso.

Sin embargo, existen discrepancias entre la intencionalidad de liderar de los Jefes de Unidad y la percepción de los enfermero/as, específicamente respecto a las metodologías utilizadas para promover un clima participativo, generar posibilidades de desarrollo y gestionar la comunicación de modo eficaz. A partir de las entrevistas, se observa que los métodos utilizados por los jefes son diferentes, con escasa integración de intervenciones entre ellos y carece de un criterio unificado alineado a una visión estratégica de la gestión de personas.

A modo consecuente, los enfermeros perciben el ejercer de los jefes como discrecional siendo que gran parte de sus reflexiones resultan de observar lo que dice y hace el jefe. De acuerdo con un relevamiento reciente, la consistencia de los líderes entre lo que dicen y lo que hacen, la participación de los empleados en un lugar psicológica y emocionalmente saludable para trabajar son algunas de las características que distinguen a las mejores empresas para trabajar y aquello que se busca en el momento de evaluar una marca empleadora (Cardozo et al., 2012).

En simultáneo, se refuerza la gestión centrada en la persona que ocupa el puesto de trabajo. Así, esta modalidad de liderar se aleja de una gestión centrada en una visión estratégica transversal, que pone el foco en el posicionamiento del rol del líder.

Finalmente, la mayoría de los estudios dedicados a la rotación y retención del personal de enfermería se ha focalizado en la vinculación de este aspecto con incentivos no monetarios (autonomía laboral, desarrollo profesional, empleo flexible, elección de los turnos laborales y más días de descanso laboral), incentivos monetarios (retribuciones directas e indirectas, como salarios o sueldos, bonificaciones, pensiones, seguros, primas, becas, préstamos y reembolso de gastos de docencia) y características etarias, de género y nivel de formación académica y factores contextuales (mercado laboral, el apoyo de la familia y la ubicación del puesto de trabajo).

Visto las conclusiones pertinentemente realizadas y el 3er objetivo específico que plantea desarrollar una propuesta competitiva y sostenible de retención de Recursos Humanos basada en un modelo de Liderazgo ajustado a las necesidades de los colaboradores, en este apartado se expone una propuesta de intervención que pretende brindar herramientas y acompañamiento a los jefes en el proceso de desarrollo del liderazgo transformacional y de servicio, alineado a las expectativas organizacionales y en lo particular a las necesidades de los enfermero/as profesionales. Para tal fin, se planteó desarrollar un plan de intervención denominado Programa Elegirnos versión EP (enfermero/as 
profesionales). A continuación, se detalla la descripción de las fases:

\section{Fase 1:}

Se comenzó por generar espacios de sensibilización en relación con el propósito de abordar la problemática de la fuga de enfermero/as profesionales. Esta metodología tiene como objetivos promover la adaptación a los cambios tanto para los líderes como colaboradores, transmitir sentido de urgencia frente a la problemática de fuga de talentos, generar espacios de construcción colaborativa y de valor agregado. Como así, motivar a los líderes a apropiarse y ser parte del proceso de cambio de la cultura organizacional, pudiendo materializar y profesar el propósito. La modalidad de intervención es el taller, el cual está destinado al directorio y todos los líderes del Departamento de Enfermería; siendo los moderadores los responsables de RRHH.

\section{Fase 2:}

Si bien la Institución ya cuenta con la definición de las competencias genéricas, surge como recomendación la formalización precisa y detallada de las competencias específicas, tareas críticas, objetivos y requisitos técnicos para diseñar el perfil de puesto de enfermero/a según el área hospitalaria que desempeñe (ejemplo: Perfil enfermero/a Urgencias; Perfil enfermero/a áreas críticas).

Está fase permite sentar las bases para luego desarrollar las otras herramientas organizacionales relacionadas con la gestión del desempeño.

\section{Fase 3:}

La fase 3 se denomina gestión del desempeño dado que provee al líder diferentes herramientas relacionadas con la valoración del desempeño de los colaboradores y el feedback entre líder y colaborador.

Asimismo, está orientada a dar respuesta a la necesidad de los enfermeros/as de contar con más espacios de retroalimentación por parte del líder y, así maximizar la circulación de ideas y conocimientos en todos los niveles de la organización.
Se plantea brindar al líder un marco de evaluación del desempeño del colaborador basado en una metodología eficiente y con un criterio unificado. Esto permite lograr una mayor objetividad en el proceso de evaluación, lo cual impactará positivamente en los enfermero/as siendo que la gran mayoría visualiza al sistema de evaluación del desempeño y a los criterios de rotación interna de puestos como poco claros e incluso discrecionales. El enfermero/a tendrá una guía para saber hacia dónde orientar su desempeño de acuerdo con las expectativas del líder e institución.

Para el desarrollo del proceso de evaluación, se planteó en la fase anterior crear los perfiles de puestos según área hospitalaria. En este punto, se sugiere seguir los criterios de Cravino (2018) y se propone implementar un proceso de evaluación del desempeño basado en tres momentos temporales reflejados en un ciclo del desempeño: planeamiento, seguimiento y evaluación propiamente dicha.

A fin de acompañar a los líderes en el fortalecimiento de la gestión del desempeño, resulta de gran importancia realizar capacitaciones de entrenamiento en el proceso de evaluación y administración del instrumento y posterior retroalimentación de la misma a los colaboradores.

Otra metodología propuesta en esta fase es el programa de inducción al puesto de trabajo.

En esta línea, se considera favorable incluir desde el área de RRHH la práctica de administrar entrevistas de seguimiento con los enfermero/as en los periodos iniciales a su ingreso a la Institución. Como ya se ha expuesto, las intervenciones que desarrollan los jefes actualmente carecen de estas herramientas, al menos de manera explícita y conectada de forma directa con el objeto de estudio.

\section{Fase 4:}

Las metodologías de esta fase son desarrollar un plan de sucesión de puestos de liderazgo, trabajando con los líderes en la detección de potenciales candidatos al puesto. 
Y un plan de rotación interno de puestos según áreas hospitalarias, configurado de acuerdo los requisitos que debe contar un enfermero/a para rotar de un área a otra.

Es importante señalar que estos planes brindan a Recursos Humanos un marco para abordar el proceso de selección de cobertura de puestos críticos de manera anticipada y planificada.

\section{Fase 5:}

En esta fase, se propone generar un plan de entrenamiento de habilidades de liderazgo de tipo transformacional, de servicio y basado en la visión planteada por la inteligencia emocional. $Y$ un plan de entrenamiento de habilidades de comunicación asertiva. En simultáneo, se llevará a cabo un plan de coaching a líderes a fin de acompañarlos en su rol el cual será moderado por los responsables de RRHH.

Otra intervención es generar un programa de inducción al puesto de líder a fin de crear un marco que guíe al jefe respecto de lo que se espera de su función, adquiera progresivamente el conocimiento de sus responsabilidades y fortalezca la confianza en su rol.

En esta fase se sugieren implementar dos estrategias destinadas a estimular a los enfermero/as asistenciales a futuro ocupar puestos de jefatura, potenciar el desarrollo de las personas, estimular un ambiente colaborativo del aprendizaje y promover la formación continua solicitada por los enfermero/as. En esta línea, se propone abrir espacios académicos internos a la Institución (ejemplo: ateneos) moderados por los enfermero/as profesionales.

Por último, se propone resignificar y formalizar la práctica de acompañamiento al ingresante, el jefe de unidad deberá elegir a aquel enfermero/a apto según su desempeño y valores para ocupar el rol de coach al ingresante.

Las metodologías anteriormente expuestas de las fases 2 a la 5, serán desarrolladas según los criterios establecidos por los responsables de RRHH, consensuados con jefe y supervisor técnico del Departamento de Enfermería. Y también, se convocará al jefe de unidad de cada área.

A modo de resumen, el programa elegirnos versión $\boldsymbol{E P}$ busca ensamblar herramientas de gestión y de liderazgo bajo una metodología eficiente, sustentable, integral, estratégica y transversal a la gestión de las personas y aportar lo especifico y necesario para atraer a los enfermero/as profesionales. Dado el recorrido del presente estudio y el contexto y cultura organizacional de la Institución, se estima una favorable receptividad de las propuestas realizadas por parte de los actores estratégicos, como así también, una implementación exitosa con resultados extraordinarios. Es importante aclarar que aún el programa no se ha implementado, está en vías de aplicación para el 2022.

\section{REFERENCIAS BIBLIOGRÁFICAS}

- Anito, Oshiro y Otero (2012). Retención del capital humano valioso en consultoras globales en Argentina. Recuperado en: https://repositorio.uade.edu.ar/xmlui/handle/123456789/3904

- Batzan, P. (1987). Dirección de personal. Organización y técnicas. Barcelona, España. Editorial Hispano Europea.

- Benavides Almeida, P. (2017). La fórmula del talento. Definiciones, forma de medirlos y su integración. Palermo Business Review Palermo Business Review. Fundación Universidad de Palermo. Graduate School of Business.

- Bennis, W y Nanus, B. (2008). Líderes. Estrategias para un liderazgo eficaz. España: Paidos.

- Borracchia, C. (2016). Los jóvenes no sueñan con compañías. Por qué debés gestionar tu marca empleadora. Buenos Aires, Argentina: Nicolás Agustín Di Candia.

- Branham, W. (2010). The new HR Analytics de Jac Fitz Enz. Recuperado en: https:// repositorio.uade.edu.ar/xmlui/bitstream/handle/123456789/5257/GRANJA. pdf?sequence=1\&isAllowed=y

- Cardozo, Barash, Bocci, Cravino, Arteche, Lanfranco Rio. (2012). Desarrollo Humano en 
las Organizaciones. Buenos Aires, Argentina: Temas.

- Castello R., Garbancho L., Scafidi H. y Morlegan L. (2008).Capital Humano: Una mirada crítica sobre un futuro complejo. Buenos Aires, Argentina: Edicon.

- Castillo, S. (2009). Nuevas tendencias en la retención y mejora del talento profesional y directivo. España: Fundación EOI.

- Chiavenato, I. (2007). Administración de RRHH. DF, México. McGraw-Hill/Interamericana editores, S.A.

- Chiavenato, I. (2007). Administración de RRHH. DF, México. McGraw-Hill/Interamericana editores, S.A.

- Chiavenato, I. (2009). Gestión del talento.

DF, México: Mc GRAW HILL Educación.

- Chiavenato, I. (2000). Administración de Recursos Humanos. Bogotá: Mc GRAW-HILL INTERAMERICANA.

- CIE (1987). Definición de enfermera. Recuperado de: https://www.icn.ch/es/politicade-enfermeria/definiciones

- CIE (2006). Escasez mundial de enfermeras sectores prioritarios de intervención. Informe CIE. Recuperado de: https://www.consejogeneralenfermeria.org/internacional/cie/ send/25-cie/358-14-la-escasez-mundial-deenfermeras-sectores-de-intervencin-prioritaria

- Cravino, L. (2003). Un trabajo feliz. Buenos Aires, Argentina: Editorial Temas.

- Cravino, L. (2018). Administración del Desempeño. Buenos Aires, Argentina. A.O Consulting S.A

- Cravino, L. M. (2018). Gestión del Desempeño, C.A.B.A., Argentina: Editorial Académica Español.

- Dalmau, C. y Carbonell, M. (2013). Caso de estudio de la aplicación de la Teoría de la Motivación de Pink Daniel. Recuperado de:

- Davis, K. y Newstrom, J. (2011). El comportamiento humano en el trabajo. Recuperado de: https://repository.urosario.edu.co/bitstream/handle/10336/12141/Liderazgo\%20 y\%20Cultura\%200rganizacional\%20en\%20 Latinoamerica\%20\%20Luis\%20Rivera\%20 Gonz\%C3\%A1lez.pdf?sequence=1

- Dychtwald, Erickson y Morison (2007). Retención del Talento. Madrid, España: Editorial LID.

- Estadística anual Hospital Privado de Co- munidad (2018). Mar del Plata, Argentina. Recuperado de: https://www.hpc.org.ar/hpcen-numeros/

- Estadística del OPS (2019). Recuperado de: https://www.eldia.com/ nota/2019-5-12-19-17-0-argentina-tieneuna-de-las-tasas-mas-bajas-de-la-region-encantidad-de-enfermeros-informacion-general. - Figueroa, H. (2014). Estrategias de atracción y retención del talento humano en la industria minera colombiana. Revista Económicas CUC, 35(1), 61-77.

- Furnham, A. (2011). Psicología Organizacional, el comportamiento del individuo en las organizaciones. Alfaomega.

Goleman D. (1996). Inteligencia emocional. Barcelona, España: Kairos

- Gan, F. y Soto, R. (2006). Carrera Profesional: Claves, competencias y vitaminas. Madrid :Díaz de Santos.

- Hatum, A. (2018). El anti líder. Por qué las personas no renuncian a las empresas sino a sus jefes. Buenos Aires, Argentina: Editorial: PenguinRandomHouse.

- Hatum, A. (2011). El Futuro del Talento: Gestión del talento para sobrevivir a la crisis. Buenos Aires: Temas UADE.

- Hidalgo, H. (2011). Remuneraciones inteligentes: una mirada sencilla para atraer, retener y motivar al talento. Buenos Aires, Argentina: Editorial Granica. http://epidauro. com.ar/la-situacion-de-la-enfermeria-en-argentina/ http://repositorio.usfq.edu.ec/bitstream/23000/2810/1/108747.pdf

- Jericó, P. (2011). La nueva gestión del talento. Construyendo compromiso. Madrid, España: Pearson Educación

- Jiménez, A. (2016). EmployerBranding: 14 preguntas y una conclusión. Capital humano, No 8.

- Lyle Spencer y Signe Spencer (1993). Evaluación de competencias en el trabajo. Modelos para un Desempeño Superior. Boston, Estados Unidos.

- Maslow, A. (1991). Motivación y personalidad. Madrid, España. Editorial: Harper

- Michaels, E., Axelrod B. Handfield-Jones H. (2003). La Guerra por el talento. La gestión del talento, fuente principal de ventaja competitiva. Madrid, España: Editorial Universitaria Ramón Areces.

- Mina, P. (2001). Atracción y retención del 
talento. Problemática en empresas IT Argentina. Tesis de Maestría. Universidad Blas Pascal. Buenos Aires, Argentina.

- Molina, Y. (2016). Revisión literaria del periodo 2013-2018, entorno al abordaje de cultura organizacional en organizaciones de América Latina (Tesis de pregrado). Universidad Cooperativa de Colombia, Popayán.

- Nardelli, C. F. (2018). El potencial uso del coaching ontológico en el ejercicio del Mando Militar. (Tesina de certificación como Coach Ontológico). Escuela de Formación de Líderes, C.A.B.A.

- Paradiso, G. (s/f). Organizando un proceso efectivo de feedback. Recuperado de: file:///C:/Users/Marina/Downloads/Organizando $\% 20$ un $\% 20$ proceso $\% 20$ efectivo $\% 20$ de\%20feedback.pdf

- Peña Batzan (1990). Dirección de personal. Organización y técnicas. Barcelona, España: Editorial Hispano Europea.

- Pink, D. H (2009). Drive: the surprising truth about what motivaties us. Ney York, NY: Riverhead Books.

- Polifrone, E. (2018). Recuperado en: file:///C:/Users/Marina/Downloads/Liderazgo_Transformacional_y_Liderazgo_de_Servicio.pdf

- René D. y Gonzaléz M. (2009). Estrategias de retención del personal. Una reflexión sobre su efectividad y alcances. Revista Universidad EAFIT, vol. 45, núm. , pp. 45-72

- Repiso, J. (2019). La situación de la enfermería en la Argentina. Recuperado en:

- Robbins, S. y Coulter, M (2009). Administración. Décima edición. México: Pearson Educación.

- Robbins, S., y Judge, T. (2009). Comportamiento Organizacional. México: Pearson Prentice Hall.

- Rothwell, W. (2005). Planificación de sucesión efectiva. New York, Estados Unidos: Amacom.

- Sánchez, S. (2017). Detección, atracción, selección y retención del talento en $\mathrm{RRHH}$ (Tesis Maestría). Universidad MURH ICADE, España.

- Sarasqueta, G. y otros (2010). Liderazgo y negociación. Capacidades integrales para el desempeño eficaz en contextos competitivos. Buenos Aires, Argentina: Temas Grupo Editorial.
- Schein, E. (1988). La cultura empresarial y el liderazgo. Una visión dinámica. Barcelona, España: Plaza \& Janes Editores. P.

- Solorio, R. (2012). Trabajo en equipo. Estados Unidos Mexicanos.

- Spencer, L. M. y Spencer, S. M. (s.f.). Evaluación de Competencia en el Trabajo: Modelos para un Desempeño Superior., (s.l.): (s.n.).

- Stegmann, T; Troncoso, R. y Gallardo, V. (2001) Proposición de un Modelo de Evaluación de Desempeño para la Gestión Actual de los Recursos Humanos tesis para optar al Grado de Magíster en Administración y Dirección de Recursos Humanos. Santiago de Chile, Chile: USACH.

- Watzlawick P, Beavin J. y Jackson D. (1991). Teoría de la comunicación humana. Barcelona, España: Herder.

- Zurn, Dolea y Stilwell (2006). Contratación y retención de las enfermeras: formación de unos recursos humanos motivados. La iniciativa de análisis mundial de enfermería. Ginebra, Suiza. 